\title{
Ashwagandha root extract exerts anti-inflammatory effects in HaCaT cells by inhibiting the MAPK/NF-кB pathways and by regulating cytokines
}

\author{
ABUDUBARI SIKANDAN, TAKAHISA SHINOMIYA and YUKITOSHI NAGAHARA \\ Division of Life Science and Engineering, School of Science and Engineering, Tokyo Denki University, \\ Hatoyama, Saitama 350-0394, Japan
}

Received November 18, 2017; Accepted March 29, 2018

DOI: $10.3892 / \mathrm{ijmm} .2018 .3608$

\begin{abstract}
A paste composed of the boiled leaves and roots of the Ashwagandha plant is used to cure ulcer and swelling in Ayurvedic medicine. However, the effects of the hot water extract of Ashwagandha roots (ASH-WEX), which is also used in Ayurveda, on skin have not been fully elucidated. Therefore, the present study investigated the anti-inflammatory activity of ASH-WEX on skin, by using the human keratinocyte cell line $\mathrm{HaCaT}$. The results indicated that ASH-WEX significantly inhibited mRNA expression of inflammatory cytokines, including interleukin (IL)-8, IL-6, tumor necrosis factor (TNF- $\alpha$ ), IL-1 $\beta$ and IL-12, and promoted the mRNA expression of the anti-inflammatory cytokine transforming growth factor (TGF)- $\beta 1$ in HaCaT cells. In addition, ASH-WEX inhibited the lipopolysaccharide-induced phosphorylation of p38 and c-Jun N-terminal kinase, as well as the nuclear translocation of nuclear factor (NF)- $\mathrm{B}$ p65. Downregulation of TNF- $\alpha$ mRNA and upregulation of TGF- $\beta 1$ mRNA were also observed in vivo following ASH-WEX treatment of mouse skin. In conclusion, the present study demonstrated that the anti-inflammatory effect of ASH-WEX may be due to its ability to suppress the NF- $\kappa \mathrm{B}$ and mitogen-activated protein kinase pathways, and to modulate cytokine expression. These results suggest that ASH-WEX can potentially protect against skin inflammation.
\end{abstract}

Correspondence to: Professor Yukitoshi Nagahara, Division of Life Science and Engineering, School of Science and Engineering, Tokyo Denki University, Hatoyama, Hiki-gun, Saitama 350-0394, Japan

E-mail: yuki@mail.dendai.ac.jp

Abbreviations: TNF, tumor necrosis factor; IL, interleukin; ASH-WEX, Ashwagandha root water extract; LPS, lipopolysaccharide; JNK, c-Jun N-terminal kinase; ERK, extracellular signal-regulated

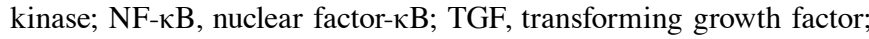
$\mathrm{PCR}$, polymerase chain reaction; MAPK, mitogen-activated protein kinase

Key words: Ashwagandha, anti-inflammation, mitogen-activated protein kinase, nuclear factor- $\kappa \mathrm{B}$, cytokine modulation

\section{Introduction}

Human skin is divided into epidermal, dermal and fatty subcutaneous tissues. The epidermis, which is comprised mainly of keratinocytes, is classified into multiple layers, the stratum corneum, granular layer, spinous layer and basal layer, based on the differentiation stage of the keratinocytes (1). Keratinocytes are known to have an important role in inflammation (2). Indeed, many inflammation-related skin diseases, such as allergic contact dermatitis, psoriasis and atopic dermatitis, are highly associated with the function of keratinocytes and cytokines (3). Keratinocytes are also a major source of inflammatory mediators, including members of the tumor necrosis factor (TNF)- $\alpha$ and interleukin (IL) families (2). Overproduction of pro-inflammatory mediators may lead to an abnormal inflammatory response. For this reason, potential anti-inflammatory agents can be used to suppress pro-inflammatory mediators (4).

Multiple studies have discovered anti-inflammatory and wound healing activities in substances extracted from plants $(5,6)$. The effects of Ashwagandha (Withania Somnifera) leaves and roots, one of the most popular herbal treatments in the traditional Ayurvedic medicine in India, are considered to include enhancements in physical strength, energy, resistance to various stresses, and immunity. Since the roots of the Ashwagandha plant are also known to be beneficial in treating ulcers, leucoderma and scabies, they have also been applied topically to heal skin sores and reduce swelling (7). These findings suggest that one or more components of Ashwagandha have active physiological effects on the skin. Studies on topical treatment with Ashwagandha organic and aqueous extracts have confirmed their chemopreventive effects on skin cancer (8) and their melanin regulatory effects (9). In addition, Ashwagandha, in the form of a paste of boiled leaves and roots, is considered to have wound-healing abilities in Ayurveda (10).

It has been determined that the main organic solvent extract of Ashwagandha is the water-insoluble Withaferin-A, and that Withaferin-A exhibits anti-inflammatory, antiangiogenesis, antimetastasis and anticancer activities (11). In Ayurveda, however, most herbal remedies are applied as a hot-water extract. Whether the healing effects of 
Withaferin-A would be observed by applying a hot-water extract of Ashwagandha (ASH-WEX) to skin remains largely unexplored.

Recently, a report determined that ASH-WEX had physiological activities and effects that were different than those of Withaferin-A, and were unrelated to Withaferin-A (12). Bhat et al (13) also reported that Ashwagandha tea increased natural killer-cell activity, possibly due to various effects of the components of the tea in cytokine secretion. The present study hypothesized that ASH-WEX may modulate the expression of cytokines when used as a topical treatment for skin. Thus, the anti-inflammatory effects and the intracellular mechanisms of ASH-WEX were investigated in vitro and in vivo.

\section{Materials and methods}

Materials. Ashwagandha roots were a kind gift from Dr Reiji Nishio (Toray Industries, Inc., Tokyo, Japan). Lipopolysaccharide (LPS) and MTT were purchased from Wako Pure Chemical Industries, Ltd. (Osaka, Japan). Primary antibodies for p38 (cat. no. 9212S), phosphorylated (P)-p38 (cat. no. 9211S), c-Jun N-terminal kinase (JNK) (cat. no. 9252S), P-JNK (cat. no. 9251S), extracellular signal-regulated kinase (ERK) (cat. no. 9102S), P-ERK (cat. no. 9101S),

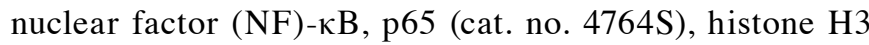
(cat. no. 9715) and $\beta$-actin (cat. no. 4967S) were purchased from Cell Signaling Technology, Inc. (Danvers, MA, USA). TNF- $\alpha$ polyclonal antibody (bs-2081R) was purchased from Bioss Antibodies, Inc. (Woburn, MA, USA). TGF- $\beta 1$ polyclonal antibody (cat. no. 5559-100) was purchased from BioVision, Inc. (Milpitas, CA, USA). Horseradish peroxidase-conjugated goat anti-rabbit IgG (cat. no. 7074) (Cell Signaling Technology, Inc.) was used as a secondary antibody. Medatomidine hydrochloride and atipamezole hydrochloride were purchased from Wako Pure Chemical Industries, Ltd. Vetorphale was purchased from Meiji Seika Kaisha, Ltd. (Tokyo, Japan) and midazolam was purchased from Sandoz (Tokyo, Japan).

Extraction of ASH-WEX. Several root pieces from different Ashwagandha roots were cut and powdered with ceramic mortar, and then added to Milli Q water $(5 \mathrm{~g}$ powder in $50 \mathrm{ml}$ water). After $2 \mathrm{~h}$ of heating with an electric heater (Tokyo Technological Laboratories, Tokyo, Japan) at $100^{\circ} \mathrm{C}$, the extract was passed through a paper filter (GE Healthcare Life Sciences, Little Chalfont, UK). Following centrifugation at $180 \mathrm{x} \mathrm{g}$ for $5 \mathrm{~min}$, the supernatants were filtered with a $0.2 \mu \mathrm{m}$ filter (Sartorius AG, Goettinggen, Germany) and stored at $-80^{\circ} \mathrm{C}$ until use. The final concentration of ASH-WEX was $100 \mathrm{mg} / \mathrm{ml}$. ASH-WEX was diluted with Dulbecco's modified Eagle medium (DMEM; Sigma-Aldrich; Merck KGaA, Darmstadt, Germany) or Milli Q prior to its use in the treatment of $\mathrm{HaCaT}$ cells or mouse skin, respectively.

Cell culture. The human keratinocyte cell line HaCaT was provided by Professor P. Boukamp and Dr N. Fusenig (German Cancer Research Center, Heidelberg, Germany) (14) and cultured in DMEM supplemented with $10 \%$ fetal bovine serum (FBS; Biofill, Victoria, Australia), $100 \mathrm{U} / \mathrm{ml}$ penicillin and $100 \mu \mathrm{g} / \mathrm{ml}$ streptomycin at $37^{\circ} \mathrm{C}$ in a humidified chamber under an atmosphere of $95 \%$ air and $5 \% \mathrm{CO}_{2}$.

Animals. A total of 18 six-week-old male C57BL/6J mice (weight, $\sim 20 \mathrm{~g}$ ) were purchased from Tokyo Laboratory Animals Science Co., Ltd. (Tokyo, Japan). The mice were given ad libitum access to standard chow and water, and housed in our departmental animal room under specific pathogen-free conditions. All animals were kept in a controlled environment for 1 week prior to experiments and all experimental procedures were approved by the Tokyo Denki University Animal Care and Use Committee in accordance with their Ethical and Animal Experiment Regulations.

Cell viability assay. Cell viability was evaluated by MTT assay. $\mathrm{HaCaT}$ cells $\left(1 \times 10^{4}\right.$ cells/well) were cultured in 96-well plates for $24 \mathrm{~h}$ (Thermo Fisher Scientific, Inc., Waltham, MA, USA) and then treated with different concentrations of ASH-WEX for $24 \mathrm{~h}$. Cells were then incubated with $10 \mu \mathrm{l}$ of $5 \mathrm{mg} / \mathrm{ml}$ MTT for $1 \mathrm{~h}$ at $37^{\circ} \mathrm{C}$. Finally, the supernatants were discarded and DMSO (100 $\mu \mathrm{l} /$ well) was added to dissolve MTT formation. The absorbance was measured with a microplate reader (Awareness Technology, Palm City, FL, USA) at $570 \mathrm{~nm}$.

$R N A$ isolation and reverse transcription-quantitative polymerase chain reaction (RT-qPCR) in vitro. HaCaT cells $\left(1 \times 10^{6}\right.$ cells/dish) were incubated in a dish (diameter, $\left.100 \mathrm{~mm}\right)$ for $24 \mathrm{~h}$ and then treated with ASH-WEX $(0.25$ or $1,5 \mathrm{mg} / \mathrm{ml})$ for $24 \mathrm{~h}$, and total RNA was isolated by TRIzol (Thermo Fisher Scientific, Inc.) according to the manufacturer's protocol. PrimeScript Reverse Transcriptase (Takara Bio, Inc., Kusatsu, Japan) was used for the reverse transcription reaction. In brief, $2.5 \mu \mathrm{l}$ of Milli Q, $2 \mu \mathrm{l}$ of $5 \mathrm{X}$ PrimeScript buffer, $2 \mu \mathrm{l}$ of $2.5 \mathrm{mM}$ dNTP, $2 \mu \mathrm{l}$ of $2.5 \mu \mathrm{M}$ Oligo dT (Sigma-Aldrich; Merck KGaA), $0.25 \mu \mathrm{l}$ of PrimeScript, $0.25 \mu 1$ of recombinant RNase inhibitor and $1 \mu 1$ of isolated RNA $(1 \mu \mathrm{g} / \mu \mathrm{l})$ were combined as a single sample, incubated at $42^{\circ} \mathrm{C}$ for $30 \mathrm{~min}$ and $70^{\circ} \mathrm{C}$ for $15 \mathrm{~min}$, and placed in short-term storage at $4^{\circ} \mathrm{C}$ in a thermal cycler (ASTEC, Shime, Japan). Reverse transcriptase-generated complementary DNA encoding IL-1 $\beta$, IL-6, IL-8, IL-12, TNF- $\alpha$, and transforming growth factor (TGF)- $\beta 1$ genes were amplified by PCR using the following specific primers: IL- $1 \beta$ forward, GCTGAGGAAGATGCT GGTTC and reverse, TCCATATCCTGTCCCTGGAG; IL-6 forward, CCTGAACCTTCCAAAGATGGC and reverse, TTC ACCAGGCAAGTCTCCTCA; IL-8 forward, GTCCTTGTT CCACTGTGCCT and reverse, GCTTCCACATGTCCTCAC AA; IL-12 forward, GATGGCCCTGTGCCTTAGTA and reverse, TCCATATCCTGTCCCTGGAG; TNF- $\alpha$ forward, TCCTTCAGACACCCTCAACC and reverse, TCCTTCAGA CACCCTCAACC; TGF- $\beta$ forward, CCCTGGACACCA ACTATTGC and reverse, GTCCAGGCTCCAAATGTAGG; and GAPDH forward, ATCATCAGCAATGCCTCCTG and reverse, GTGCTTCACCACCTTCTTGA. In brief, a reaction solution [12.5 $\mu \mathrm{l}$ of $2 \mathrm{X}$ GeneAce SYBR qPCR Mix $\alpha$ (Wako Pure Chemical Industries, Ltd.), $0.5 \mu 1$ of each primer, Milli Q, and $2.5 \mu \mathrm{l}$ of cDNA sample] was dispensed into optical 96-well reaction plates (Applied Biosystems; Thermo Fisher Scientific, Inc.), and set in an ABI PRISM 7000 (Applied Biosystems; Thermo Fisher Scientific, Inc.). Then, amplification consisted 
of 1 cycle at $95^{\circ} \mathrm{C}$ for $10 \mathrm{~min}$, followed by 45 cycles at $95^{\circ} \mathrm{C}$ for $30 \mathrm{sec}$ and $60^{\circ} \mathrm{C}$ for $1 \mathrm{~min}$, with a final dissociation stage at $95^{\circ} \mathrm{C}$ for $15 \mathrm{sec}, 60^{\circ} \mathrm{C}$ for $20 \mathrm{~min}$, and $95^{\circ} \mathrm{C}$ for $15 \mathrm{sec}$. Relative fold changes in mRNA expression were calculated using the $2^{-\Delta \Delta \mathrm{Cq}}$ method, according to Livak and Schmittgen (15).

Western blot analysis. HaCaT cells ( $1 \times 10^{6}$ cells/dish) were incubated in a dish (diameter, $100 \mathrm{~mm}$ ) for $24 \mathrm{~h}$ and then pretreated with ASH-WEX $(5 \mathrm{mg} / \mathrm{ml})$ for $20 \mathrm{~h}$, followed by $4 \mathrm{~h}$ incubation with LPS $(1 \mu \mathrm{g} / \mathrm{ml})$. To examine the mitogen-activated protein kinase (MAPK) pathway, total proteins were extracted with lysis buffer (50 mM HEPES, $150 \mathrm{mM} \mathrm{NaCl}, 10 \%$ glycerol, $1 \%$ Triton-X 100, $1.5 \mathrm{mM} \mathrm{MgCl}_{2}, 1 \mathrm{mM}$ EGTA, $1 \mathrm{mM}$ sodium orthovanadate, $1 \%$ protease inhibitor cocktail; $\mathrm{pH} 7.5$ ) and western blot was performed as previously described (16). To investigate the $\mathrm{NF}-\kappa \mathrm{B}$ translocation to the nucleus, the nuclear fractions were prepared as previously described (17) with slight modifications. HaCaT cells $\left(1 \times 10^{6}\right.$ cells/dish) were incubated a dish (diameter, $100 \mathrm{~mm}$ ) for $24 \mathrm{~h}$ and then pretreated with ASH-WEX for $20 \mathrm{~h}$, followed by $4 \mathrm{~h}$ incubation with LPS $(1 \mu \mathrm{g} / \mathrm{ml})$. After the cells were collected and washed with PBS, the pellets were resuspended in $100 \mu \mathrm{l}$ of buffer A [10 mM HEPES, 10 mM KCl, 0.1 mM EDTA, 0.4\% (v/v) NP-40, 1\% protease inhibitor; $\mathrm{pH}$ 7.9] and incubated on ice for $10 \mathrm{~min}$. Following centrifugation at 15,000 x g for $3 \mathrm{~min}$, the supernatant was collected as a cytosolic fraction. The remaining pellets were washed with buffer A, resuspended in $60 \mu \mathrm{l}$ of buffer B (20 mM HEPES, 0.4 M NaCl, 1 mM EDTA, 10\% glycerin, $1 \%$ protease inhibitor; $\mathrm{pH} 7.9$ ) and stirred at $4^{\circ} \mathrm{C}$ for $2 \mathrm{~h}$. The supernatants were collected as a nucleic fraction following centrifugation at $15,000 \mathrm{x}$ g at $4^{\circ} \mathrm{C}$ for $3 \mathrm{~min}$. Protein concentrations were determined using a BCA protein assay kit (Thermo Fisher Scientific, Inc.). For each sample, $20 \mu \mathrm{g}$ of protein was subjected to $12 \%$ polyacrylamide gel electrophoresis, and then transferred to a polyvinylidene difluoride membrane (Bio-Rad Laboratories, Inc., Hercules, CA, USA). The membranes were incubated in blocking solution (3\% non-fat skimmed milk) for $1 \mathrm{~h}$, and then probed with primary antibodies $(1: 1,000)$ at $4^{\circ} \mathrm{C}$ overnight. After washing with $0.1 \%$ Tween 20 -PBS, the membrane was incubated with secondary antibody $(1: 2,000)$ for $1 \mathrm{~h}$ at room temperature. Finally, the membranes were visualized with enhanced chemiluminescence reagents (Pierce ECL Western Blotting Substrate; Thermo Fisher Scientific, Inc.) following exposure for $1 \mathrm{~min}$, and observed with an Image Quant LAS-4000 system (GE Healthcare Life Sciences). The relative density of bands was quantified using ImageJ software (National Institutes of Health, Bethesda, MD, USA).

RNA isolation, RT-qPCR and wound-healing effect of $A S H$-WEX in vivo. The anti-inflammatory effect of ASH-WEX in vivo was investigated using 7-week-old male C57BL/6J mice. The back of each mouse was shaved under anesthesia (mixed anesthesia of $0.75-\mathrm{mg} / \mathrm{kg}$ bw medatomidine hydrochloride, $4-\mathrm{mg} / \mathrm{kg}$ bw midazolam, and $5-\mathrm{mg} / \mathrm{kg}$ bw vetorphale in physiological saline and antagonist of $0.75-\mathrm{mg} / \mathrm{kg}$ bw atipamezole hydrochloride in physiological saline) (18), and a $0.5-\mathrm{cm}$ diameter wound area was excised on both sides of the back using a dermal punch (Nipro, Osaka, Japan), for initiation of an inflammatory response. Each mouse was topically treated with Milli Q on one side ( $20 \mu \mathrm{l}$; control side) and

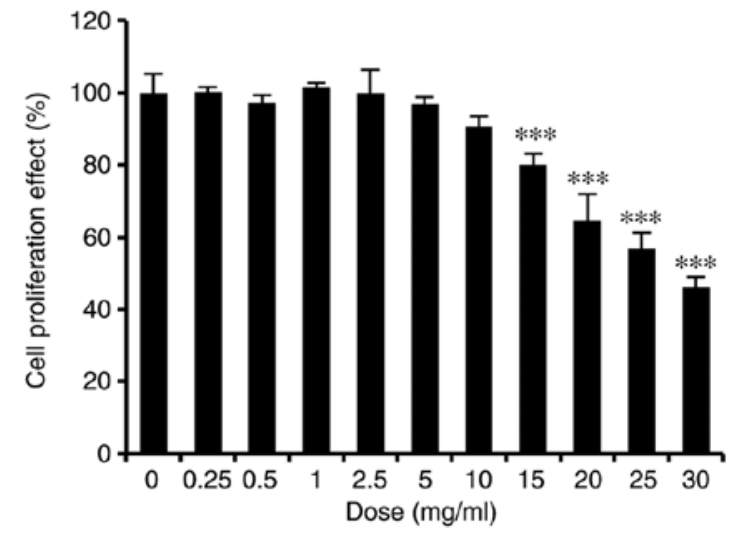

Figure 1. Effect of ASH-WEX on cell viability. HaCaT cells were treated with the indicated concentration of ASH-WEX for $24 \mathrm{~h}$. Cell viability was assessed by MTT assay. The quantified data are shown as the mean \pm standard deviation of three independent experiments. ${ }^{* * * *} \mathrm{P}<0.001$ vs. untreated cells. ASH-WEX, Ashwagandha root water extract.

with ASH-WEX on the other side $(20 \mu \mathrm{l} ; 10 \mathrm{mg} / \mathrm{ml}$; treatment side). Following treatment, two types of tape (Tegaderm Roll 16006S, 3M Health Care, Tokyo, Japan; Dressing tape, Kyowa, Osaka, Japan) were placed at the sites , in order to avoid direct contact with the wound and to prevent evaporation. The tapes were peeled off gently and changed following topical treatment. Each mouse was housed separately, and treated every $24 \mathrm{~h}$. The day when the wounds were made and treated with ASH-WEX or Milli Q was designated as day 0. The wounds were scanned and photographed prior to treatment and every day, until day 11. Wound closure was calculated as follows: wound closure of Day $n=$ Day $n$ wound area/Day 0 wound area. The weight of each mouse was measured every day following treatment, with a Table Top High Precision Balance (A\&D Company, San Jose, CA, USA). Relative wound closure, expressed as the wound size ratio of day n/day 0 , was quantified using ImageJ software.

To investigate cytokine expression, mice were sacrificed on day 5 , and a $1 \times 1 \mathrm{~cm}$ area of injured skin was removed and used for RNA extraction. Total RNA was isolated by TRIzol (Thermo Fisher Scientific, Inc.) according to the manufacturer's protocols. Following reverse transcription, RT-qPCR was performed, as aforementioned. The following primers were used: TNF- $\alpha$ forward, CCACCACGCTCTTCTGTCTA and reverse, CAC TTGGTGGTTTGCTACGA; TGF- $\beta$ forward, TTGCTTCAG CTCCACAGAGA and reverse, TGGTTGTAGAGGGCAAGG AC; and GAPDH forward, AACTTTGGCATTGTGGAAGG and reverse, ACACATTGGGGGTAGGAACA.

Hematoxylin and eosin $(H \& E)$ staining. Seven-week-old male C57BL/6J mice were treated with ASH-WEX or Milli Q as described above. On day 5, six mice were sacrificed and a $1 \times 1 \mathrm{~cm}$ area including the entire wound and surrounding unwounded skin were collected for H\&E staining. Mouse skin samples were fixed in $4 \%$ paraformaldehyde at $4^{\circ} \mathrm{C}$ for $72 \mathrm{~h}$. The samples were dehydrated and embedded in paraffin and skin sections were cut at a thickness of $5 \mu \mathrm{m}$. Following deparaffinization and rehydration, the samples were stained with $\mathrm{H} \& \mathrm{E}$ and the epidermal and dermal thicknesses were measured by ImageJ. Digital images were captured with an 

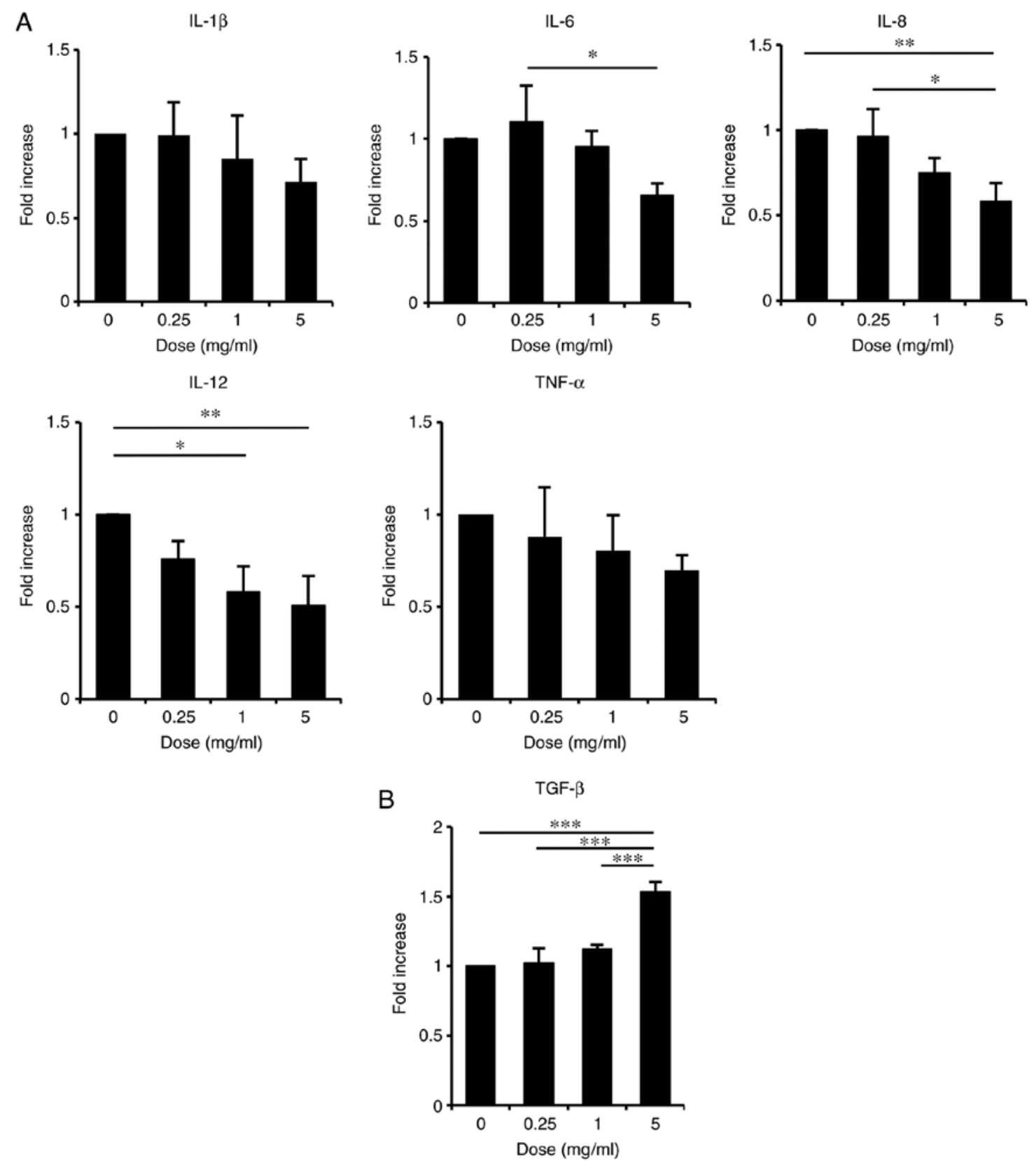

Figure 2. Differential expression of cytokine mRNA in ASH-WEX-treated HaCaT cells. HaCaT cells were treated with the indicated concentration of ASH-WEX for $24 \mathrm{~h}$, then the mRNA expression levels of (A) pro-inflammatory and (B) anti-inflammatory cytokines were analyzed. The quantified data are shown as the mean \pm standard deviation of three independent experiments. GAPDH was used as a control. ${ }^{*} \mathrm{P}<0.05,{ }^{* *} \mathrm{P}<0.01$ and ${ }^{* * * *} \mathrm{P}<0.001$ with comparisons indicated by lines. ASH-WEX, Ashwagandha root water extract; IL, interleukin; TNF, tumor necrosis factor; TGF, transforming growth factor.

Olympus BX51 microscope (Olympus Corporation, Tokyo, Japan) and DP714 digital camera (Olympus Corporation).

Statistical analysis. The Student's t-test was used to compare paired groups. One-way analysis of variance was used for multi-group analysis, followed by Bonferroni test as a post hoc test (Prism 7, ver. 7.0d, GraphPad Software, Inc., La Jolla, CA, USA). $\mathrm{P}<0.05$ was considered to indicate a statistically significant difference.

\section{Results}

Low toxicity effect of ASH-WEX on HaCaT cells. Cells of the human keratinocyte line HaCaT have features similar to normal human cells, and thus are widely applied in inflammatory skin-related research. In the present study, $\mathrm{HaCaT}$ cells were used to investigate the anti-inflammatory effects of ASH-WEX. To determine whether ASH-WEX may have a toxic effect on HaCaT cells, an MTT assay was performed. The results demonstrated that ASH-WEX was not toxic to $\mathrm{HaCaT}$ cells up to a dose of $10 \mathrm{mg} / \mathrm{ml}$ (Fig. 1).

ASH-WEX treatment downregulates inflammatory cytokines, while it upregulates anti-inflammatory cytokines. Cytokines released from keratinocytes have vital roles in many inflammation-related skin diseases (19). In order to examine whether ASH-WEX might regulate the cytokine expression in keratinocytes, the expression levels of several cytokines 

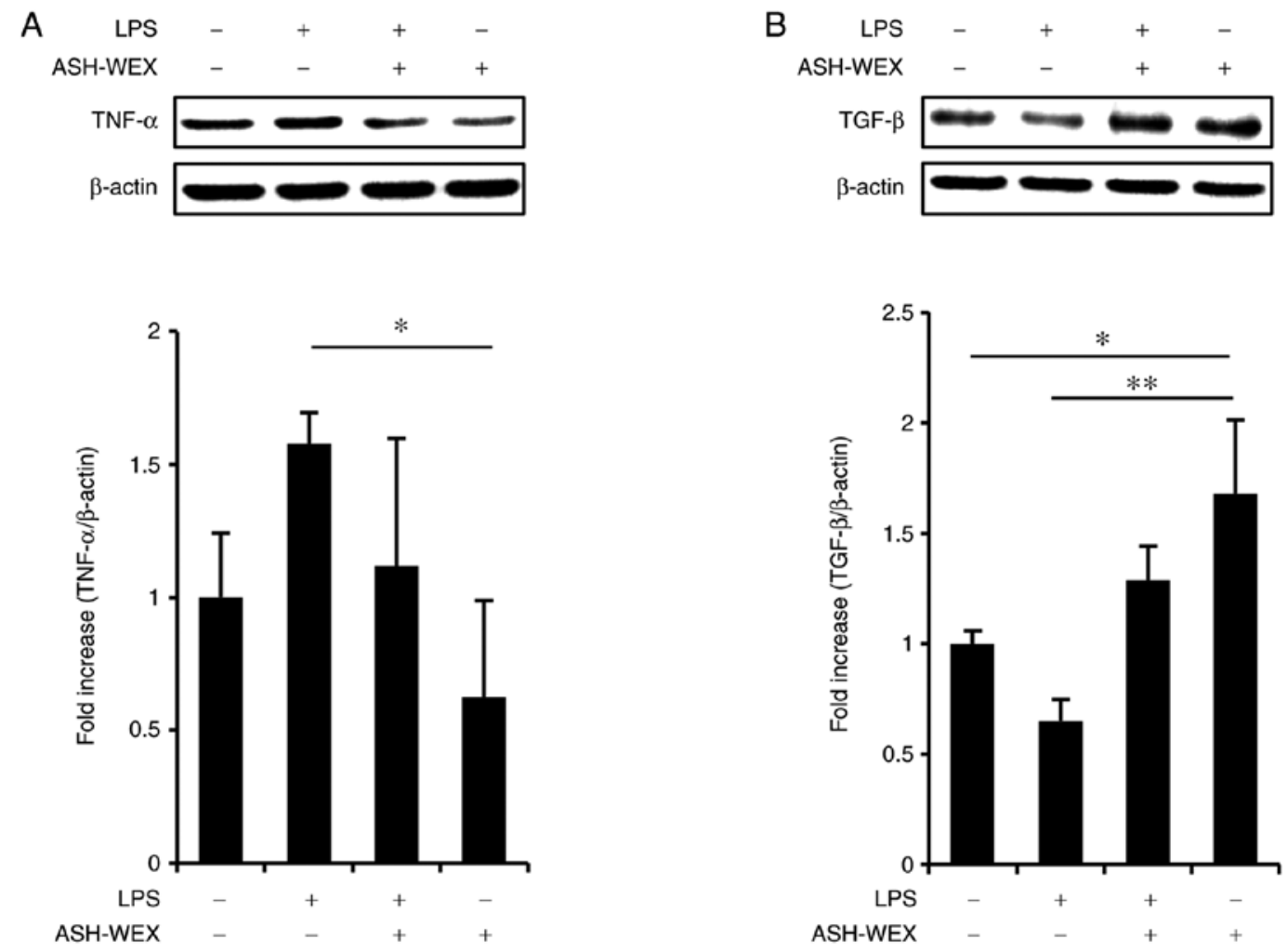

Figure 3. Differential expression of cytokine protein in ASH-WEX-treated HaCaT cells. HaCaT cells were treated with $5 \mathrm{mg} / \mathrm{ml}$ of ASH-WEX for $12 \mathrm{~h}$ and then with LPS ( $1 \mu \mathrm{g} / \mathrm{ml}$ ) for $12 \mathrm{~h}$. (A) TNF- $\alpha$ and (B) TGF- $\beta$ protein expression levels were determined by western blot analysis. The relative density of the TNF- $\alpha$, TGF- $\beta$ and $\beta$-actin bands were estimated and the mean \pm standard deviation of three independent experiments are presented as quantified data." $\mathrm{P}<0.05$ and ${ }^{* *} \mathrm{P}<0.01$ with comparisons indicated by lines. ASH-WEX, Ashwagandha root water extract; LPS, lipopolysaccharide; TNF, tumor necrosis factor; TGF, transforming growth factor.

were examined in HaCaT cells treated with ASH-WEX by RT-qPCR. The results demonstrated that, when cells were treated with ASH-WEX at concentrations $<5 \mathrm{mg} / \mathrm{ml}$ which were non-toxic in HaCaT cells (Fig. 1), the mRNA expression levels of the inflammatory cytokines IL-8, IL-6 and IL-12 were significantly decreased in a dose-dependent manner (Fig. 2A). The mRNA levels of TNF- $\alpha$ and IL-1 $\beta$ also appeared decreased, although this effect was less significant (Fig. 2A). By contrast, treatment with ASH-WEX at concentrations $<5 \mathrm{mg} / \mathrm{ml}$ significantly increased the mRNA expression levels of the anti-inflammatory cytokine TGF- $\beta 1$ in a dose-dependent manner (Fig. 2B). These results indicated that ASH-WEX might have an anti-inflammatory effect on keratinocytes.

$\mathrm{HaCaT}$ cells possess Toll-like receptor (TLR) 2 and TLR4 (20), and the expression of pro-inflammatory cytokines, including IL-8, IL-6, TNF- $\alpha$, IL-1 $\beta$ and IL-12, are modulated by TLR signaling (21). Therefore the TLR4 agonist, LPS, was used as a model of inflammation and the effects of ASH-WEX cytokine production were investigated at the protein level by western blotting. The results demonstrated that treatment with $5 \mathrm{mg} / \mathrm{ml} \mathrm{ASH-WEX} \mathrm{inhibited} \mathrm{the} \mathrm{LPS-induced} \mathrm{TNF-} \alpha$ upregulation (Fig. 3A), but significantly increased TGF- $\beta 1$ levels (Fig. 3B). These results were in accordance with the mRNA results (Fig. 2).

ASH-WEX treatment inhibits the MAPK/NF- $\kappa B$ pathways. The present data suggested that ASH-WEX might have anti-inflammatory properties by suppressing the expression of inflammatory cytokines. It is well stablished that the NF- $\mathrm{KB}$ and MAPK signaling pathways are strongly associated with inflammatory cytokine expression in HaCaT cells $(22,23)$. LPS stimulation activates NF- $\mathrm{kB}$ by modulating TLR4 (24). LPS can also activate the MAPK pathway in HaCaT cells (22). To determine whether ASH-WEX suppressed the LPS-induced MAPK and NF- $\kappa \mathrm{B}$ pathways, a western blot analysis was performed. The results revealed that treatment with $5 \mathrm{mg} / \mathrm{ml}$ ASH-WEX inhibited the LPS-induced phosphorylation of p38 (Fig. 4A) and JNK (Fig. 4B), but upregulated the phosphorylation of ERK (Fig. 4C). Next, it was investigated whether ASH-WEX inhibited LPS-induced nuclear transduction of NF- $\mathrm{kB}$, and the results demonstrated that treatment with $5 \mathrm{mg} / \mathrm{ml} \mathrm{ASH-WEX}$ inhibited the LPS-induced nuclear translocation of p65 (Fig. 4D), which is a significant mediator of the NF- $\kappa B$ signaling pathway.

Effect of ASH-WEX on cytokine expression and wound-closure effect in vivo. Finally, the anti-inflammatory effect of ASH-WEX was investigated in vivo. It is well known that external stimuli, such as wounds, initiate inflammation in the skin (25). In the present study, wounded mouse skin was employed as an in vivo inflammation model in order to investigate the anti-inflammatory effects of ASH-WEX. On each mouse, ASH-WEX was topically applied to a wound on the left side of the back skin and solvent Milli Q was topically applied to a wound at the right side of the back skin every $24 \mathrm{~h}$ until 

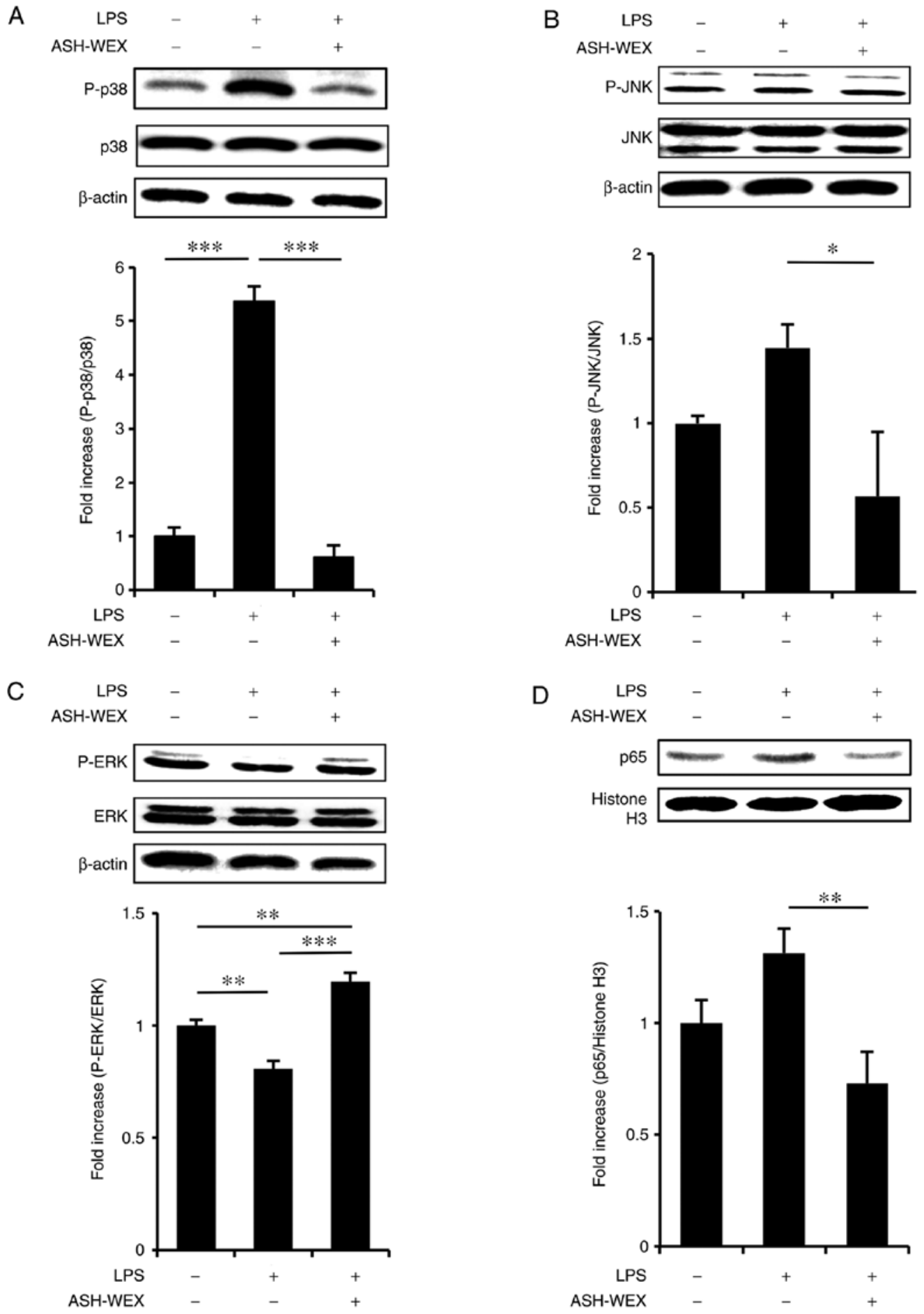

Figure 4. ASH-WEX treatment attenuates the LPS-induced inflammatory response signaling. HaCaT cells $\left(1 \times 10^{5}\right.$ cells $)$ were treated with $5 \mathrm{mg} / \mathrm{ml}$ of ASH-WEX for $20 \mathrm{~h}$ and then with LPS (1 $\mu \mathrm{g} / \mathrm{ml})$ for $4 \mathrm{~h}$. (A) The levels of p38 MAPK phosphorylation, (B) JNK phosphorylation, and (C) ERK phosphorylation were measured by western blotting. $\beta$-actin was used as internal control. (D) The protein levels of p65 NF-kB in the nuclear fraction of the treated cells were determined by western blotting. Histone $\mathrm{H} 3$ was used as internal control. The relative density of the bands was estimated and the mean \pm standard deviation of three independent experiments is presented as quantified data. ${ }^{*} \mathrm{P}<0.05,{ }^{* * *} \mathrm{P}<0.01$ and ${ }^{* * * *} \mathrm{P}<0.001$ with comparisons indicated by lines. ASH-WEX, Ashwagandha root water extract; LPS, lipopolysaccharide; MAPK, mitogen-activated protein kinase; JNK, c-Jun N-terminal kinase; ERK, extracellular signal-regulated kinase; $\mathrm{NF}-\kappa \mathrm{B}$, nuclear factor- $\mathrm{KB} ; \mathrm{P}-$, phosphorylated.

day 5. Thereafter, the skin was removed and used to assay the mRNA expression levels of cytokines by RT-qPCR. The results demonstrated that ASH-WEX treatment inhibited the mRNA expression of the pro-inflammatory cytokine TNF- $\alpha$, while it increased the mRNA expression of the anti-inflammatory cytokine TGF- $\beta 1$ (Fig. 5). These results further demonstrated that
ASH-WEX may regulate the inflammatory response associated with wound closure (26).

Finally, a wound-healing assay was performed in vivo to examine the properties of ASH-WEX. The results demonstrated that, on days 1-5, the ASH-WEX treatment side exhibited a significantly decreased wound area compared 
A

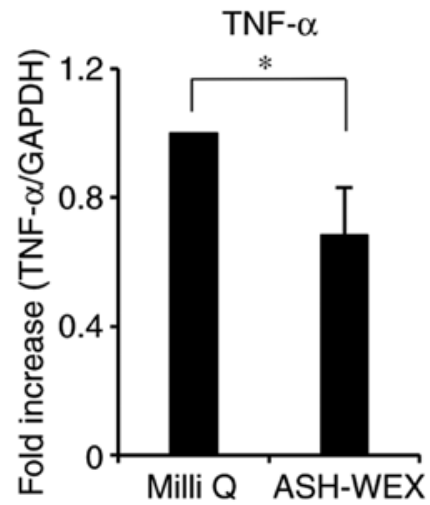

B

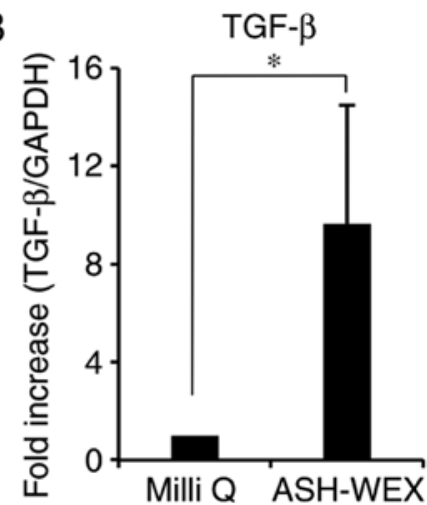

Figure 5. Differential expression of cytokine mRNAs in ASH-WEX-treated mouse skin. The back of each mouse was shaved and a $0.5 \mathrm{~cm}$ diameter wound area was excised on both sides of the back. Mice were treated with Milli Q on one side and ASH-WEX on the other, once every $24 \mathrm{~h}$. Mice were sacrificed, and the RNA was isolated on day 5. (A) TNF- $\alpha$ and (B) TGF- $\beta$ mRNA expressions were analyzed by reverse transcription-quantitative polymerase chain reaction. The quantified data are shown as the mean \pm standard deviation of three independent experiments. GAPDH was used as a control. "P<0.05. ASH-WEX, Ashwagandha root water extract; TNF, tumor necrosis factor; TGF, transforming growth factor.

A
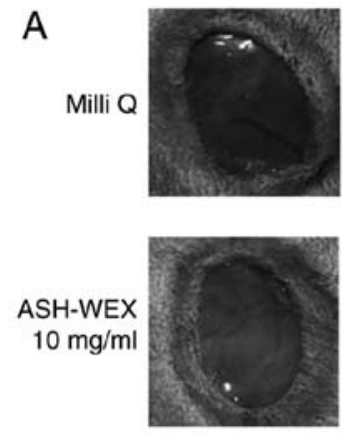

Days
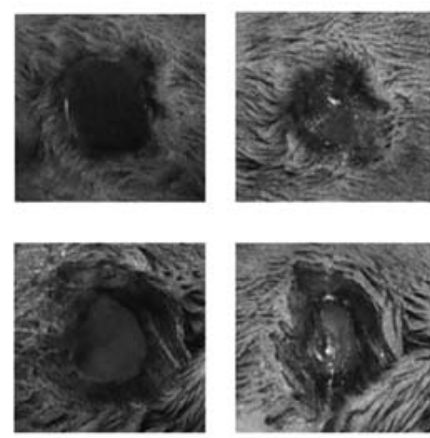

2

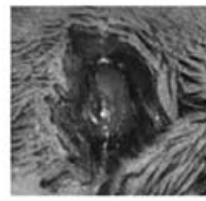

4
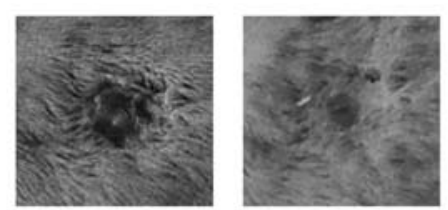

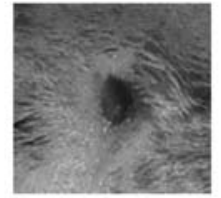

6

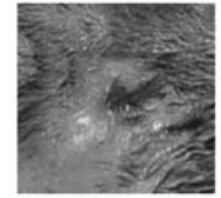

8
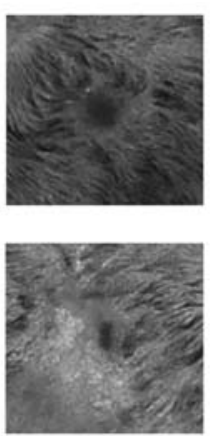

10

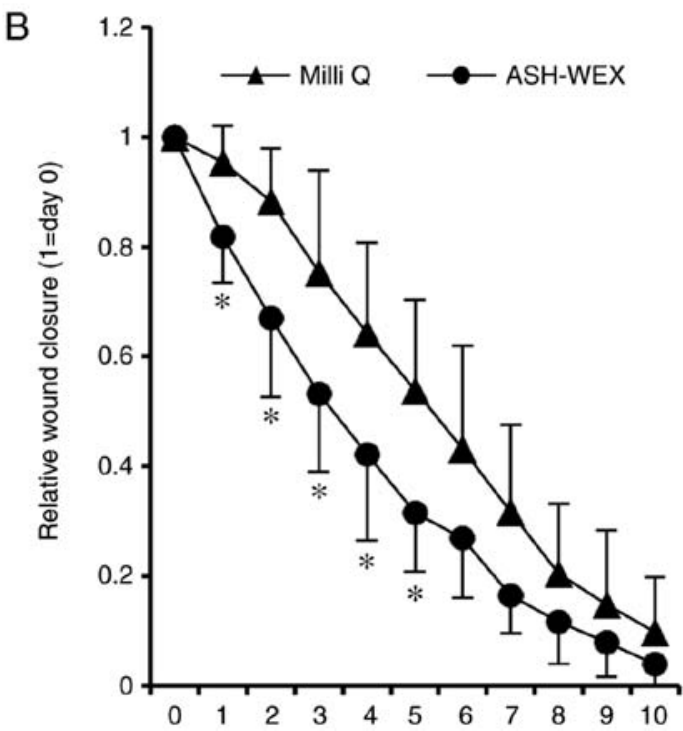

Figure 6. Effect of ASH-WEX treatment on wound closure in mice. The back of each mouse was shaved and a wound area $0.5 \mathrm{~cm}$ in diameter was excised on both sides of the back. The wounds were treated with Milli Q on one side and ASH-WEX on the other, once every 24 h. (A) Representative photographs of wound sites from six independent experiments. (B) Quantification of wound area per treatment group is shown as the mean \pm standard deviation of six independent experiments. ${ }^{*} \mathrm{P}<0.05$ vs. the control-treated side. ASH-WEX, Ashwagandha root water extract.

with the control Milli Q- treated side (Fig. 6). These results suggest that ASH-WEX treatment accelerated the speed of wound-closure in the early stage, which is the inflammation phase of wound healing. In addition, the ASH-WEX-treated skin displayed a thinner epidermis and thinner dermis compared with the control skin (Fig. 7). Furthermore, less aggregation of immune cells was observed in the ASH-WEX-treated skin compared with the control-treated skin (Fig. 7). Of note, ASH-WEX treatment did not affect the weight of the mice (data not shown). 

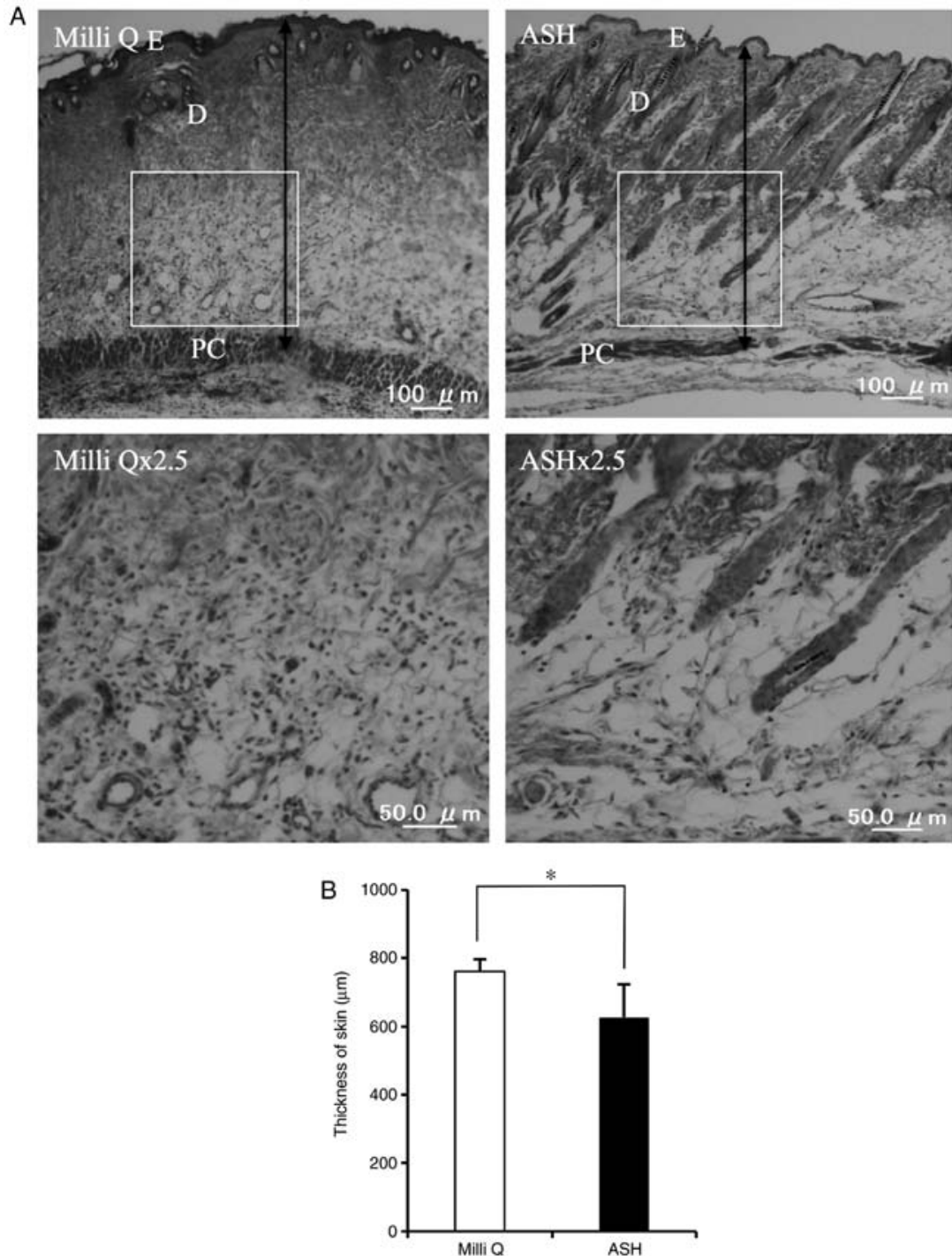

Figure 7. Histological changes in the wounded mouse skin following ASH-WEX treatment. The back of each mouse was shaved and a $0.5 \mathrm{~cm}$ diameter wound area was excised on both sides of the back. Mice were treated with Milli Q on one side and ASH-WEX on the other, once every $24 \mathrm{~h}$. The skin wound samples were collected and stained with H\&E and the epidermal and dermal thickness were measured by ImageJ software. (A) Representative images of H\&E-stained skin sections from the Milli Q-treated (Milli Q) or ASH-WEX-treated (ASH) wound area. The lower images represent a 2.5x enlargement of the boxed areas of the corresponding upper images. Black-arrowed lines indicate the thickness of skin. (B) Quantified data of the thickness of skins are shown as the mean \pm standard deviation of four independent experiments. ${ }^{*} \mathrm{P}<0.05$ vs. the control-treated side. ASH-WEX, Ashwagandha root water extract $\mathrm{D}$, dermis; E, epidermis; PC, panniculus carnosus muscle.

\section{Discussion}

The present study investigated the anti-inflammatory and cytokine modulatory effect of ASH-WEX in vitro. HaCaT cells were used to examine the cytokine modulatory effect of ASH-WEX, and the results revealed that ASH-WEX treatment inhibited the MAPK and NF- $\mathrm{KB}$ pathways to decrease the expression of pro-inflammatory cytokines and increase the expression of anti-inflammatory cytokines. Finally, ASH-WEX treatment was demonstrated to also exert an anti-inflammatory effect in vivo.

The present results indicated that ASH-WEX treatment resulted in a decrease of pro-inflammatory cytokine expression in HaCaT cells at both the mRNA and protein level. To investigate the mechanism underlying these effects, the MAPK pathway and its downstream pathway NF- $\kappa \mathrm{B}$ were investigated, which are strongly related to pro-inflammatory cytokine release in monocytes (27). Ultraviolet electromagnetic radiation (UV) and bacteria can activate the MAPK and NF- $\kappa \mathrm{B}$ pathways $(4,28)$. Tang et al (4) confirmed that the anti-inflammatory effect of glycolic acid is due to its ability to inhibit UVB-induced mRNA expression of inflammatory cytokines and NF- $\kappa \mathrm{B}$ nuclear translocation in $\mathrm{HaCaT}$ cells. In addition, LPS can activate NF- $\kappa \mathrm{B}$ and MAPK signaling in $\mathrm{HaCaT}$ cells $(20,21)$. In the present study, LPS-treated $\mathrm{HaCaT}$ cells were used to determine whether ASH-WEX suppressed the LPS-induced MAPK and NF- $\mathrm{BB}$ pathways. The results demonstrated that ASH-WEX indeed inhibited the LPS-induced phosphorylation of the MAPKs p38 and JNK, as well as the nuclear translocation of p65 NF- $\kappa$ B. These results are in agreement with the study of Wang et al (29), in which the anti-inflammatory agent Baicalein inhibited LPS-induced p38 MAPK and NF- $\kappa B$ activation. There has been at least one report that Ashwagandha roots exert an antibacterial 
effect (30). Based on the present results, ASH-WEX may also have antibacterial activity.

Multiple plants possess wound-healing activity due to the anti-inflammatory effects of active components in their extracts (31). It is well known that external stimuli, such as wounds, initiate inflammation in the skin. Indeed, one of the main and early phases of wound healing is the inflammatory phase, in which many pro-inflammatory mediators, such as TNF- $\alpha$, are released in the wound area to initiate inflammation (32). This inflammatory response is indispensable for wound repair. However, an excessive or prolonged inflammation phase will delay wound healing (33). TGF- $\beta 1$ serves a vital role in wound healing, and increased levels of TGF- $\beta 1$ signal the transition from inflammatory phase to proliferation phase, which is another essential stage of wound healing (34). In the present study, at days 1-5 following ASH-WEX treatment, the size of the wound decreased significantly compared with the control-treated wound. Furthermore, histological analysis revealed that the immune cells were less aggregated in the ASH-WEX-treated skin compared with the control skin. These data suggested that the control side remained in the inflammatory phase, while the ASH-WEX-treated side rapidly progressed to the proliferation phase. Ghlissi et al (35) suggested that the wound-healing activity of Artemisia campestris aqueous extract in vivo may be due to the reduction of the inflammatory phase, and the rapid transition to the proliferation phase by its anti-inflammatory effect. Collectively, these findings suggest that ASH-WEX may be a promising wound-healing agent, and thus the wound-healing activity of ASH-WEX warrants further investigation.

Both the organic and the aqueous extract of Ashwagandha have anti-inflammatory activity $(36,37)$. Previous phytochemical studies have confirmed that Ashwagandha contains alkaloids, flavonols, withanolides, glycowithanolides, steroidal lactones, sterols and phenolic acids (7,38-40). The most bioactive ingredients from the root include sitoindosides (VII, VIII, IX, X) withanine, withananine, and ashwagandhanolide (41). However, most of the bioactive components of Ashwagandha have been isolated from its organic layer extracts. There are few studies about the bioactive ingredients of ASH-WEX. One of the existing reports on this subject indicates that the main component of ASH-WEX is Withaferin-A (42), but, when the solubility of purified Withaferin-A in water was tested by heating for $2 \mathrm{~h}$, the same condition used to extract ASH-WEX, the Withaferin-A powder remained in the solution. Another study reported that 5 withanolides and one glyco-withanolide were the active constituents of ASH-WEX (43). Withanolides have anti-inflammatory activities (44). To the best of our knowledge, the anti-inflammatory effects of withanolides on $\mathrm{HaCaT}$ cells remain unclear. Recently, a study reported that triethylene glycol (TEG) was present in the aqueous extract of Ashwagandha, and particularly concentrated in the leaves (12). In the present study, therefore, we tested whether TEG modulates the levels of cytokine expression in $\mathrm{HaCaT}$ cells and revealed that TEG suppresses the LPS-induced expression of the inflammatory cytokines TNF- $\alpha$, IL-1 $\beta$, IL-6 and IL-8 (data not shown). TEG also inhibited LPS-induced nuclear transduction of NF- $\mathrm{NB}$ (data not shown). From this result, TEG might be one of the bioactive components of ASH-WEX that induce anti-inflammation. However, the anti-inflammatory effects of TEG and its wound-healing activities require further investigation.

In conclusion, the present study demonstrated that ASH-WEX had an anti-inflammatory effect, which it exerted by suppressing the NF- $\mathrm{B}$ and MAPK pathways, and by modulating the cytokine expression in HaCaT cells. ASH-WEX treatment increased the mRNA expression of the anti-inflammatory cytokine TGF- $\beta 1$ and decreased the mRNA expression of the pro-inflammatory cytokine TNF- $\alpha$ in vivo. Thus, ASH-WEX could be a promising anti-inflammatory agent for skin, as well as a potential wound-healing therapeutic agent.

\section{Acknowledgements}

We are grateful to Mr. Daiki Yagi for technical assistance.

\section{Funding}

No funding was received.

\section{Availability of data and materials}

The analyzed datasets generated during the study are available from the corresponding author on reasonable request.

\section{Author's contributions}

AS, TS and YN designed the experiments; AS performed the experiments. AS and YN collected and analysed data. TS critically revised the manuscript for intellectual content. AS and YN wrote the manuscript. All authors read and approved the final manuscript.

\section{Ethics approval and consent to participate}

All experimental procedures were approved by the Tokyo Denki University Animal Care and Use Committee in accordance with their Ethical and Animal Experiment Regulations.

\section{Consent for publication}

Not applicable.

\section{Competing interests}

The authors declare that they have no competing interests.

\section{References}

1. McGrath JA, Eady RAJ and Pope FM: Anatomy and organization of human skin, In: Burns T, Breathnach S, Cox N and Griffiths C (eds). Rook's Textbook of Dermatology. 7th edition. Blackwell Publishing Inc., Malden, pp3.1-3.84, 2004.

2. Juránová J, Franková J and Ulrichová J: The role of keratinocytes in inflammation. J Appl Biomed 15: 169-179, 2017.

3. Albanesi C, Scarponi C, Giustizieri ML and Girolomoni G: Keratinocytes in inflammatory skin diseases. Curr Drug Targets Inflamm Allergy 4: 329-334, 2005

4. Tang SC, Liao PY, Hung SJ, Ge JS, Chen SM, Lai JC, Hsiao YP and Yang JH: Topical application of glycolic acid suppresses the UVB induced IL-6, IL-8, MCP-1 and COX-2 inflammation by modulating NF- $\kappa \mathrm{B}$ signaling pathway in keratinocytes and mice skin. J Dermatol Sci 86: 238-248, 2017. 
5. Kim M, Lee HJ, Randy A, Yun JH, Oh SR and Nho CW: Stellera chamaejasme and its constituents induce cutaneous wound healing and anti-inflammatory activities. Sci Rep 7: 42490, 2017.

6. Ghlissi Z, Kallel R, Sila A, Harrabi B, Atheymen R, Zeghal K, Bougatef A and Sahnoun A: Globularia alypum methanolic extract improves burn wound healing process and inflammation in rats and possesses antibacterial and antioxidant activities. Biomed Pharmacother 84: 1488-1495, 2016.

7. Kurian A and Asha Sankar M: Medicinal Plants. Peter KV (ed.). New India Publishing, New Delhi, pp61-68, 2007.

8. Prakash J, Gupta SK and Dinda AK: Withania somnifera root extract prevents DMBA induced squamous cell carcinoma of skin in swiss albino mice. Nutr Cancer 42: 91-97, 2002.

9. Imokawa $\mathrm{G}$ and Ishida $\mathrm{K}$ : Inhibitors of intracellular signaling pathways that lead to stimulated epidermal pigmentation: Perspective of anti-pigmenting agents. Int J Mol Sci 15 8293-8315, 2014

10. Beena Sharma: Health awareness in women with the use of traditional medicinal plants in district raipur of Chhattisgarh. J Ecobiotech 3: 15-17, 2011.

11. Kanika P, Singh RB and Dinesh KP: Pharmacological and analytical aspects of withaferin A: A concise report of current scientific literature. Asian Pac J Reprod 2: 238-243, 2013.

12. Wadhwa R, Singh R, Gao R, Shah N, Widodo N, Nakamoto T, Ishida Y, Terao K and Kaul SC: Water extract of Ashwagandha leaves has anticancer activity: Identification of an active component and its mechanism of action. PLoS One 8: e77189, 2013.

13. Bhat J, Damle A, Vaishnav PP, Albers R, Joshi M and Banerjee G: In vivo enhancement of natural killer cell activity through tea fortified with Ayurvedic herbs. Phytother Res 24: 129-135, 2010

14. Boukamp P, Petrussevska RT, Breitkreutz D, Hornung J, Markham A and Fusenig NE: Normal keratinization in a spontaneously immortalized aneuploid human keratinocyte cell line. J Cell Biol 106: 761-771, 1988.

15. Livak KJ and Schmittgen TD: Analysis of relative gene expression data using real-time quantitative PCR and the 2(-Delta Delta C(T)) method. Methods 25: 402-408, 2001.

16. Suita H, Shinomiya T and Nagahara Y: Caspase- 6 induces 7A6 antigen localization to mitochondria during FAS-induced apoptosis of Jurkat cells. Anticancer Res 37: 1697-1704, 2017

17. Tomar D, Sripada L, Prajapati P, Singh R, Singh AK and Singh R Nucleo-cytoplasmic trafficking of TRIM8, a novel oncogene, is involved in positive regulation of TNF induced NF- $\mathrm{KB}$ pathway. PLoS One 7: e48662, 2012.

18. Kawai S, Takagi Y, Kaneko S and Kurosawa T: Effect of three types of mixed anesthetic agents alternate to ketamine in mice. Exp Anim 60: 481-487, 2011.

19. Hänel KH, Cornelissen C, Lüscher B and Baron JM: Cytokines and the skin barrier. Int J Mol Sci 14: 6720-6745, 2013.

20. Pivarcsi A, Koreck A, Bodai L, Széll M, Szeg C, Belso N, Kenderessy-Szabó N, Bata-Csörgo Z, Dobozy A and Kemény A: Differentiation-regulated expression of Toll-like receptors 2 and 4 in HaCaT keratinocytes. Arch Dermatol Res 296: 120-124, 2004.

21. Su SB, Silver PB, Grajewski RS, Agarwal RK, Tang J, Chan CC and Caspi RR: Essential role of the MyD88 pathway, but nonessential roles of TLRs 2, 4, and 9, in the adjuvant effect promoting Th1-mediated autoimmunity. J Immunol 175: 6303-6310, 2005.

22. Lv F, Yu Y, Wang G, Hu J, Zhang B, You W and Wang J: Mechanisms by which the N-terminal 24 amino acids of the p55 regulatory subunit of phosphatidylinositol 3-kinase affect endotoxin-induced cytokine release in human keratinocytes. Mol Med Rep 11: 3753-3759, 2015.

23. Wang Y, Zhang H,Du G, Wang Y, Cao T, Luo Q, Chen J, Chen F and Tang G: Total glucosides of paeony (TGP) inhibits the production of inflammatory cytokines in oral lichen planus by suppressing the NF- $\mathrm{B}$ signaling pathway. Int Immunopharmacol 36: 67-72, 2016.

24. Ge Y, Xu Y, Sun W, Man Z, Zhu L, Xia X, Zhao L, Zhao Y and Wang $X$ : The molecular mechanisms of the effect of Dexamethasone and Cyclosporin A on TLR4/NF- $\kappa B$ signaling pathway activation in oral lichen planus. Gene 508: 157-164, 2012.

25. Gurtner GC, Werner S, Barrandon Y and Longaker MT: Wound repair and regeneration. Nature 453: 314-321, 2008.
26. César I, Paggiaro AO, Conduta JL, Aldunate B, Herson MR, Altran SC, Mathor MB and Ferreira MC: Role of keratinocytes in wound contraction: An impact assessment using a model of collagen matrix populated with fibroblasts. Revista Brasileira de Cirurgia Plástica 26: 402-406, 2011.

27. Muthusamy V and Piva TJ: The UV response of the skin: A review of the MAPK, NF-kappaB and TNF-alpha signal transduction pathways. Arch Dermatol Res 302: 5-17, 2010.

28. Chung WO and Dale BA: Innate immune response of oral and foreskin keratinocytes: Utilization of different signaling pathways by various bacterial species. Infect Immun 72: 352-358, 2004.

29. Wang J, Luo H, Yang L and Li Y: Baicalein induces apoptosis and reduces inflammation in LPS-stimulated keratinocytes by blocking the activation of NF- $\kappa B$ : Implications for alleviating oral lichen planus. Cell Mol Biol (Noisy-le-grand) 62: 55-60, 2016.

30. Girish KS, Machiah KD, Ushanandini S, Harish Kumar K, Nagaraju S, Govindappa M, Vedavathi $M$ and Kemparaju K: Antimicrobial properties of a non-toxic glycoprotein (WSG) from Withania somnifera (Ashwagandha). J Basic Microbiol 46: 365-374, 2006

31. Budovsky A, Yarmolinsky L and Ben-Shabat S: Effect of medicinal plants on wound healing. Wound Repair Regen 23: 171-183, 2015.

32. Spiekstra SW, Breetveld M, Rustemeyer T, Scheper RJ and Gibbs S: Wound-healing factors secreted by epidermal keratinocytes and dermal fibroblasts in skin substitutes. Wound Repair Regen 15: 708-717, 2007.

33. Yamanaka O, Kitano-Izutani A, Tomoyose $\mathrm{K}$ and Reinach PS: Pathobiology of wound healing after glaucoma filtration surgery. BMC Ophthalmol 15 (Suppl 1): 157, 2015.

34. Zhang F, Wang $H$, Wang X, Jiang G, Liu H, Zhang G, Wang H, Fang R, Bu X, Cai S and Du J: TGF- $\beta$ induces M2-like macrophage polarization via SNAIL-mediated suppression of a pro-inflammatory phenotype. Oncotarget 7: 52294-52306, 2016.

35. Ghlissi Z, Sayari N, Kallel R, Bougatef A and Sahnoun Z: Antioxidant, antibacterial, anti-inflammatory and wound healing effects of Artemisia campestris aqueous extract in rat. Biomed Pharmacother 84: 115-122, 2016.

36. Gupta A and Singh S: Evaluation of anti-inflammatory effect of Withania somnifera root on collagen-induced arthritis in rats. Pharm Biol 52: 308-320, 2014.

37. Pawar P, Gilda S, Sharma S, Jagtap S, Paradkar A, Mahadik K, Ranjekar P and Harsulkar A: Rectal gel application of Withania somnifera root extract expounds anti-inflammatory and muco-restorative activity in TNBS-induced inflammatory bowel disease. BMC Complement Altern Med 11: 34, 2011

38. Singh P, Guleri R, Singh V, Kaur G, Kataria H, Singh B, Kaur G, Kaul SC, Wadhwa R and Pati PK: Biotechnological interventions in Withania somnifera (L.) Dunal. Biotechnol Genet Eng Rev 31: $1-20,2015$.

39. Trivedi MK, Panda P, Sethi KK and Jana S: Metabolite profiling in Withania somnifera roots hydroalcoholic extract using LC/MS, GC/MS and NMR spectroscopy. Chem Biodivers 14, 2017.

40. Alam N, Hossain M, Khalil MI, Moniruzzaman M, Sulaiman SA and Gan SH: High catechin concentrations detected in Withania somnifera (ashwagandha) by high performance liquid chromatography analysis. BMC Complement Altern Med 11: 65, 2011.

41. Mill BA, Khazir J, Mir NA, Hasan TU and Koul S: Botanical, chemical and pharmacological review of Withania somnifera (Indian Ginseng): An ayurvedic medicinal plant. Indian J Drugs Dis 1: 147-160, 2012.

42. Halder B, Singh S and Thakur SS: Withania somnifera root extract has potent cytotoxic effect against human nalignant nelanoma cells. PLoS One 10: e0137498, 2015.

43. Bani S, Gautam M, Sheikh FA, Khan B, Satti NK, Suri KA, Qazi GN and Patwardhan B: Selective Th1 up-regulating activity of Withania somnifera aqueous extract in an experimental system using flow cytometry. J Ethnopharmacol 107: 107-115, 2006.

44. Sun GY, Li R, Cui J, Hannink M, Gu Z, Fritsche KL, Lubahn DB and Simonyi A: Withania somnifera and its withanolides attenuate oxidative and inflammatory responses and up-regulate antioxidant responses in BV-2 microglial cells. Neuromolecular Med 18: 241-252, 2016. 